\title{
PRÁTICAS DE EDUCAÇÃO EM SAÚDE FRENTE AOS PORTADORES DE DIABETES MELLITUS EM UMA UBS: relato de experiência
}

\author{
Vinicius Lino de SOUZA NETO ${ }^{1}$ \\ Nathália Araújo da NÓBREGA ${ }^{2}$ \\ Gabrielly de Carly Pereira de CARVALHO ${ }^{3}$ \\ Renata Laís da Silva NASCIMENTO ${ }^{4}$ \\ Géssica Raíssa Francisco FRANÇA ${ }^{5}$ \\ José JUSTINO FILHO**
}

\begin{abstract}
${ }^{1}$ Discente do Curso Bacharelado em Enfermagem da Universidade Federal de Campina Grande e Bolsita do Programa de Extensão - PROBEX / Vigência - 2013 - UFCG; Líder do Grupo de pesquisa em Epidemiologia e Saúde Coletiva GPESC. E-mail: vinolino@ hotmail.com
\end{abstract}

${ }^{2}$ Discente do Curso Bacharelado em Enfermagem da Universidade Federal de Campina Grande e Membro do Grupo de pesquisa em epidemiologia e saúde coletiva - GPESC - UFCG; Colaborada do projeto de Extensão PROBEX/ Vigência -2013.E-mail: nathaliaanobrega@hotmail.com

${ }^{3}$ Discente do Curso Bacharelado em Enfermagem da Universidade Federal de Campina Grande e Membro do Grupo de pesquisa em epidemiologia e saúde coletiva - GPESC - UFCG; Colaborada do projeto de Extensão PROBEX/ Vigência - 2013 E-mail: gabriellycarly@gmail.com

${ }^{4}$ Discente do Curso Bacharelado em Enfermagem da Universidade Federal de Campina Grande e Membro do Grupo de pesquisa em epidemiologia e saúde coletiva - GPESC - UFCG; Colaborada do projeto de Extensão PROBEX/ Vigência - 2013 E-mail: renata-blink@hotmail.com

${ }^{5}$ Discente do Curso Bacharelado em Enfermagem da Universidade Federal de Campina Grande e Membro do Grupo de pesquisa em epidemiologia e saúde coletiva - GPESC - UFCG; Colaborada do projeto de Extensão PROBEX/ Vigência - 2013. E-mail: g.raissa.fran@gmail.com

** Coordenador do Projeto de Extensão - PROBEX/ Vigência- 2013, intitulado "Práticas de Educação em Saúde frente aos portadores de diabetes mellitus assistidos nas UB'S, zona urbana de Cuité: Uma promoção da Saúde.”. Mestrado e Doutorado em Sociologia pela Universidade Federal da Paraíba. Professor Adjunto IV do Curso de Enfermagem da Universidade Federal de Campina Grande - UFCG/ Campus- Cuité, Paraíba (PB), Brasil. E-mail: josejustino@bol.com.br

**Endereço para correspondência do autor:

Sitio olho D'água da bica, S\N, CEP: 58.175-000 Tel: 3372-1900. Professor do Curso de Graduação em Enfermagem da Universidade Federal de Campina Grande - UFCG/ Campus - Cuité.

\section{Recebido em: 12/09/2013 - Aprovado em: 14/12/2013 - Disponibilizado em: 15/01/2014}

RESUMO - Objetivo: Relatar a vivência das praticas de educação em saúde dos estudantes de enfermagem frente aos portadores de diabetes mellitus. Metodologia: Trata-se de um estudo de natureza qualitativa e descritiva, do tipo relato de experiência, das vivências dos discentes de Enfermagem no processo de articulação das praticas de educação em saúde aos diabéticos em uma unidade básica de saúde. Resultados e Discussão: Desta forma, a experiência foi vivenciada pelos colaboradores de um projeto de extensão em uma unidade básica do município de Cuité- PB, voltado ao portador de diabetes ao qual teve como mecanismo de articulação as pratica de educação em saúde, este singelo encontro foi dividido em três momentos, como: na primeira ocasião mesurou a glicemia dos participantes, consecutivamente explanou sobre a morbidade da patologia e por fim foi proporcionado um café da manha saudável, ou seja, a ideia foi de articular um dia saudável aos portadores de diabetes, sempre respeitado as suas singularidades e crenças. Conclusão: Desta forma, a pratica de educação em saúde torna-se um instrumento de transformação do meio ao qual está circunscrito o portador, respeitando sempre a sua singularidade, crenças e saberes populares. É notório que a pratica de educação em saúde é um dos vários pilares organizacionais da atenção primária a saúde.

Descritores: Educação em saúde. Diabetes Mellitus. Atenção primária a saúde. Cuidado. Enfermagem em saúde comunitária. 


\title{
PRACTICES OF HEALTH EDUCATION FRONT TO HOLDERS OF DIABETES MELLITUS IN A UBS: experience report
}

\begin{abstract}
Objective: To report the experience of the practice of health education of nursing students against diabetes mellitus. Methodology: This was a qualitative study and descriptive account of the type of experience, the experiences of nursing students in the process of articulation of the practice of health education to diabetic patients in a primary care unit. Results and Discussion: In this way, the experience was lived by employees of an extension project at a primary municipality of Cuité-PB, aimed at people with diabetes which had the hinge mechanism of the health education practice, this simple meeting was divided into three stages, as on the first occasion mesurou glycemia participants consecutively expounded on the morbidity of the disease and was eventually provided a healthy breakfast, ie, the idea was to articulate a healthy day to patients with diabetes, always respected their singularities and beliefs. Conclusion: Thus, the practice of health education becomes an instrument of transformation through which the carrier is limited, always respecting their uniqueness, beliefs and popular knowledge. It is clear that the practice of health education is one of several organizational pillars of primary health care.
\end{abstract}

Keywords: Health education. Diabetes Mellitus. Primary Health Care. Care. Community health nursing.

\section{INTRODUÇÃO}

Dentro do contexto das doenças crônicas não transmissíveis (DCNT) a diabetes mellitus vem ganhando destaque no cenário nacional, decorrente da sua incidência, a mesma caracteriza por ser uma enfermidade de fundo metabólico, ao qual afeta vários órgãos, especialmente olhos, rins, nervos, cérebro, coração e vasos sanguíneos. A sua etiologia provem da deficiência de secreção e/ou ação da insulina, envolvendo processos patogênicos específicos, como a destruição das células beta do pâncreas (produtoras de insulina), resistência à ação da insulina, distúrbios da secreção da insulina (BRASIL, 2006).

De acordo com Arsa et al, (2009) nas Américas, o número de indivíduos com diabetes foi estimado em 35 milhões para o ano 2000 e projetado para 64 milhões em 2025. Nos países desenvolvidos, o aumento ocorrerá principalmente nas faixas etárias mais avançadas, decorrente do aumento da expectativa de vida e do crescimento populacional; nos países em desenvolvimento, o aumento será observado em todas as faixas etárias, principalmente no grupo de 45-64 anos onde sua prevalência deverá triplicar, duplicando nas faixas etárias de 20-44 e 65 e mais anos.

Neste ensejo, os tipos de diabetes mais frequentes são 0 diabetes tipo 1 , anteriormente conhecido como diabetes juvenil, que compreende cerca de $10 \%$ do total de casos, e o diabetes tipo 2, anteriormente conhecido como diabetes do adulto, que compreende cerca de $90 \%$ do total de casos. Outro tipo de diabetes encontrado com maior frequência e cuja etiologia ainda não está esclarecida é o diabetes gestacional, que, em geral, é um estágio pré-clínico de diabetes, detectado no rastreamento pré-natal (BRASIL, 2009). 
As práticas de educação em saúde possibilitam um maior conhecimento sobre a patologia em questão, e favorece aos diabéticos um entendimento prático das situações. Pois a diabetes é modulada como uma doença silenciosa, ao quais os portadores tendem a negligenciar os cuidados necessários, e as práticas de educação em saúde quando conduzidas por profissionais da área qualificados, contribuem para um maior controle metabólico da diabetes no individuo, cabe aos profissionais de saúde e portador à responsabilidade de produzir as condições favoráveis ao processo de aquisição de conhecimentos sobre o DM, que possam levar à mudança nos hábitos de vida e manejo da doença (DUNCAN et al., 2004).

Desta forma, a proposta em questão tem como objetivo explanar a vivência dos extensionista do projeto, intitulado "Práticas de Educação em Saúde frente aos portadores de diabetes mellitus assistidos nas UB'S, zona urbana de Cuité: Uma promoção da Saúde”, na articulação das práticas de educação em saúde aos portadores de diabetes.

\section{METODOLOGIA}

Trata-se de um estudo de natureza qualitativa e descritiva, do tipo relato de experiência. De acordo com Lakatos e Marconi (2007), o método qualitativo difere do quantitativo não só por não empregar instrumentos estatísticos, mas também pela forma de coleta e análise dos dados. Além disso, este tipo de metodologia preocupa-se em analisar e interpretar aspectos mais profundos, descrevendo a complexidade e as peculiaridades do comportamento humano e fornece uma análise mais detalhada sobre as investigações, hábitos, atitudes e tendências do comportamento.

$$
\text { Para Gil (2008) as pesquisas }
$$
descritivas têm como objetivo primordial a descrição das características de determinada população ou fenômeno ou o estabelecimento de relações entre variáveis. No entanto, algumas pesquisas desta natureza, vão além da identificação destas variáveis, mas perpassam pela determinação da natureza dessa relação.

O presente estudo foi desenvolvido a partir de vivências acadêmicas realizadas em 1 (uma) Unidade Básica de Saúde da Família do município de Cuité - PB, no período de agosto de 2013. De acordo com dados da Secretaria Municipal de Saúde (2013) existe uma totalidade de 9 (nove) UBS's no referido município, sendo cinco na zona urbana e quatro em zona rural. As unidades que merecem maior ênfase, devido à viabilidade da realização de estudos, são localizadas na zona urbana, sendo elas: Ezequias Venâncio, Raimunda Domingos, Abílio Chacon, Diomedes Lucas e Luiza Dantas de Medeiros. Esta última serviu como campo para 
realização das praticas de educação em saúde, óbice do projeto, anteriormente citado.

Vale ressaltar que as ações desenvolvidas provêm de um projeto de extensão - PROBEX, intitulado "Práticas de Educação em Saúde frente aos portadores de diabetes mellitus assistidos nas UB'S, zona urbana de Cuité: Uma promoção da Saúde" vinculado à Universidade Federal de Campina Grande, campus Cuité - PB, sendo desenvolvido pelos discentes do curso de bacharelado em enfermagem da referida instituição.

\section{RELATO DE EXPERIÊNCIA}

A prática da experiência proporcionou aos extensionista um aprimoramento do saber e a lapidação do exercício da educação em saúde ao portador de diabetes em unidade básica de saúde. Desta forma, a vivência dos extensionista foi articulada em três momentos no lócus em questão, conforme descrito a baixo:

No primeiro momento foi realizada a mensuração da glicemia capilar dos participantes da ação, através do glicosimetro, os resultados obtidos permaneceram no limiar da mensuração em jejum (até 99 mg/dl), em seguida foi promulgada uma interlocução entre portador e extensionista, ou seja, instigou aos portadores de diabetes expor o seu saber sobre a patologia em questão, como: O que é diabetes? Quais as suas causas secundarias? Como ela afeta no desenrolar das atividades diárias?;Salientando que toda a abordagem aos mesmos foi articulada de forma simplória e enfática por uma linguagem pouco cientifica e mais coloquial.

Com isso, dentro dos discorrer das ações programadas para a unidade, logo após as primeiras intervenções educativas, conforme supracitado foi explanado ao público alvo todo o processo de contextualização da diabetes, como os seus fatores de risco e agravantes, as causas secundarias, a vigilância frente à alimentação saudável no que tange a absorção do açúcar, a importância do uso da medicação e insulina. Sendo assim, remodelamos as suas dúvidas diante das perguntas argumentando anteriormente, em seguida, foi realizada um teatro de fantoches, descrevendo um caso fictício de um paciente idoso com diabetes e não detinha o cuidado diário, onde muito dos colaboradores se identificou com o cenário exposto.

Para findar as ações, em um terceiro momento, foi servido aos diabéticos um café da manha regado a frutas e sucos, estes com baixo teor de açúcar, sendo positiva a aceitação, o momento foi marcado também pelas orientações dadas aos colaboradores pelos profissionais de saúde da presente unidade, se fez presente interinamente a enfermeira e técnico de enfermagem. Destarte, os diabéticos, colocaram em pauta que a presente ação deveria ser desenvolvida 
todo mês, para que assim fossem reciclados os seus conhecimentos sobre a enfermidade, com isso nota-se a importância da educação em saúde, em um meio que predomina a carência do conhecimento.

\section{DISCUSSÃO}

Para atingir o objetivo proposto do estudo, deve-se enfatizar o conceito de Atenção Primária à Saúde (APS). O Ministério da Saúde define APS como sendo um conjunto de práticas gerenciais e sanitárias democráticas e participativas, sob a forma de trabalho em equipe, dirigidas a populações de territórios bem delimitados, pelas quais assume a responsabilidade sanitária, considerando a dinamicidade existente no território em que vive a população (BRASIL, 2009).

Para Alves, Boehs e Heidemann (2012), o conceito de Educação em Saúde ancora-se no "conceito de Promoção da Saúde, que trata de processos que abrangem a participação de toda a população no contexto da sua vida cotidiana, não detendo-se apenas em tratar as pessoas sob o risco de adoecer". As ações educativas grupais favorecem a participação coletiva, garantindo ao indivíduo e à comunidade a possibilidade de decidirem sobre seus próprios destinos, e capacitam-os para atuarem na melhoria de sua condição vivencial. Já Rodrigues e Ribeiro (2012) conceituam educação em saúde como sendo uma maneira de interpretar as necessidades cotidianas de saúde da população, compreendendo a saúde como produção social, destacando a importância dos determinantes socioeconômicos. Essa perspectiva reafirma o compromisso político quanto à necessidade de atuar sobre os fatores biológicos, sociais, econômicos e ambientais, a fim de fomentar transformações sociais.

As práticas de educação em saúde associada a ações de promoção da saúde na Estratégia Saúde da Família (ESF) constituem um caminho integrador do cuidar, fundado em saberes técnico-científicos e culturais, capazes de promover o exercício democrático e provocar mudanças individuais, familiares e comunitárias, bem como contribuir para a transformação social (ALVES; BOEHS; HEIDEMANN, 2012).

Denota Silva et al, (2009) a diabetes mellitus é considerada como uma síndrome de acometimento universal, com um crescente quadro de morbimortalidade. Segundo o mesmo, o número de portadores da doença em todo o mundo era de 177 milhões em 2000, com expectativa de alcançar 350 milhões de pessoas em 2025. Além disso, as consequências da DM são devastadoras: são 4 milhões de mortes por ano relativas à diabetes e suas complicações, o que representa $9 \%$ da mortalidade mundial total. $\mathrm{O}$ grande impacto econômico ocorre notadamente nos serviços de saúde, como consequência dos crescentes 
custos do tratamento da doença e, sobretudo das complicações.

No cenário em que se encontra o perfil epidemiológico da população mundial, levando em consideração os crescentes índices de acometimento pela diabetes mellitus, as ações de educação em saúde configuram-se como uma estratégia primordial para controle da doença e prevenção de agravos. Para Matsumoto et al. (2012), atenção à saúde ao portador de diabetes que fornece informação oportuna, apoio e monitoramento pode melhorar a adesão aos tratamentos, reduzindo o ônus das condições crônicas e proporcionando melhor qualidade de vida às pessoas com DM.

Nesse sentido, as metas da educação em saúde em relação à doença, além de buscarem o controle da glicemia, compreendem a promoção do bem-estar da pessoa e de sua família. No entanto, as estratégias precisam ser adequadas e o conteúdo deve ser transferido de forma simples, o qual necessita ter capacidade de motivar as pessoas a compreender a doença e a assumir, de forma ativa, seu papel no tratamento, desde os aspectos mais pessoais de crenças e estado psicossocial até as implicações sociais do processo saúdedoença.

\section{CONSIDERAÇÕES FINAIS}

As modificações dos comportamentos não saudáveis presentes no estilo de vida dos diabéticos podem excluir ou controlar os fatores de risco, podendo diminuir a ocorrência, prevenir recorrências e melhorar o prognóstico dos indivíduos. A adoção de um estilo de vida saudável, associado a práticas de atividades físicas regulares e de uma alimentação saudável promove melhores padrões de qualidade de vida, o que consiste paradoxalmente em um desafio e um objetivo para este grupo populacional.

Pode-se inferir, portanto que modificações no estilo de vida devem ser instigadas a todos os pacientes hipertensos, durante todo o curso da vida. Para tanto, percebe-se que a educação em saúde é uma alternativa fundamental para conduzir esses diabéticos a essas mudanças, através de orientações que visem a melhor compreensão sobre seus hábitos e estilo de vida, para assim obter a prevenção e/ou controle dos fatores de risco da enfermidade, utilizando-se basicamente de hábitos e atitudes saudáveis.

Desta forma, a pratica de educação em saúde torna-se um instrumento de transformação do meio ao qual está circunscrito o portador, respeitando sempre a sua singularidade, ou seja, as crenças e saberes populares. É notório que a pratica de educação em saúde é um dos vários pilares organizacionais da atenção primária a saúde. 


\section{REFERÊNCIAS}

ALVES, L. H. S.; BOEHS, A. E.; HEIDEMANN, I. T. S. B. A percepção dos profissionais e usuários da estratégia de saúde da família sobre os grupos de promoção da saúde. Texto contexto - enfermagem, v. 21, n. 2, Florianópolis, 2012. Disponível: http://www.scielo.br/scielo.php?script=sci_art text\&pid=S010407072012000200019\&lng=pt\&nrm=iso Acesso em: 12 set. 2013.

ARSA, G.et al. Diabetes Mellitus tipo 2: Aspectos fisiológicos, genéticos e formas de exercício físico para seu controle. Rev. Bras.

Cineantropom Desempenho Hum, v.11, n.1, p.103-11, 2009. Disponível: http://repositorio.ucb.br/jspui/handle/1234567 89/186 Acesso em: 12 set.2013.

BRASIL. Ministério da Saúde. Secretaria de Atenção à Saúde. Departamento de Atenção Especializada. Manual de Educação em Saúde. Brasília, 2009. (Cadernos de Atenção Básica), (Série A. Normas e Manuais Técnicos). Disponível: http://bvsms.saude.gov.br/bvs/publicacoes/ma nual_educacao_saude_v2.pdf Acesso em: 12 set. 2013.

BRASIL. Ministério da Saúde. Sistema de Informação da Atenção Básica-Situação de Saúde-Paraíba. Disponível: http://tabnet.datasus.gov.br/cgi/tabcgi.exe?sia b/cnv/SIABSPB.def Acesso em: 12 de set.2013.

BRASIL. Ministério da Saúde. Secretaria de Atenção à Saúde. Departamento de Atenção
Básica. Diabetes Mellitus. Brasília, 2006. (Cadernos de Atenção Básica, 16), (Série A. Normas e Manuais Técnicos).

DUNCAN, B.B.; SCHIMIDT, M.I et al. Medicina Ambulatorial: Condutas de atenção primária baseadas em evidências. 3. ed. Porto Alegre:Artmed,2004.

GIL, A. C. Métodos e técnicas de Pesquisa Social. 6 ed. São Paulo: Atlas, 2008.

MATSUMOTO, P. M. et al. A educação em saúde no cuidado de usuários do Programa Automonitoramento Glicêmico. Rev. esc. enferm. USP, v. 46, n. 3, 2012. Disponível: http://www.scielo.br/scielo.php?script=sci_art text\&pid=S0080 62342012000300031\&lng=pt\&nrm=iso Acesso em: 13 set.2013.

LAKATOS, E. M.; MARCONI, M. de A. Fundamentos de metodologia científica. 6a.ed. São Paulo. Atlas, 2007.

RODRIGUES, C. C. ; RIBEIRO, K. S. Q. S. Promoção da saúde: a concepção dos profissionais de uma unidade de saúde da família. Trab. educ. saúde, v. 10, n. 2, 2012. Disponível:

http://www.scielo.br/scielo.php?script=sci_art text\&pid=S1981 $77462012000200004 \& \operatorname{lng}=$ pt\&nrm=iso Acesso em: 12 set. 2013.

SILVA, A. R. v et al. Educação em saúde a portadores de diabetes mellitus tipo 2: revisão bibliográfica. Rev. Rene. Fortaleza, v. 10, n. 3, p. 146-51,2009. Disponível: http://www.revistarene.ufc.br/10.3/html/17.ht m Acesso em: 12 set.2013. 